\title{
InfAcrOnt: calculating cross-ontology term similarities using information flow by a random walk
}

\author{
Liang Cheng ${ }^{1 \dagger}$, Yue Jiang ${ }^{2 \dagger}$, Hong Ju ${ }^{3}$, Jie Sun ${ }^{1}$, Jiajie Peng ${ }^{4}$, Meng Zhou ${ }^{1 *}$ and Yang $\mathrm{Hu}^{5^{*}}$
}

From 16th International Conference on Bioinformatics (InCoB 2017)

Shenzhen, China. 20-22 September 2017

\begin{abstract}
Background: Since the establishment of the first biomedical ontology Gene Ontology (GO), the number of biomedical ontology has increased dramatically. Nowadays over 300 ontologies have been built including extensively used Disease Ontology (DO) and Human Phenotype Ontology (HPO). Because of the advantage of identifying novel relationships between terms, calculating similarity between ontology terms is one of the major tasks in this research area. Though similarities between terms within each ontology have been studied with in silico methods, term similarities across different ontologies were not investigated as deeply. The latest method took advantage of gene functional interaction network (GFIN) to explore such inter-ontology similarities of terms. However, it only used gene interactions and failed to make full use of the connectivity among gene nodes of the network. In addition, all existent methods are particularly designed for $\mathrm{GO}$ and their performances on the extended ontology community remain unknown.

Results: We proposed a method InfAcrOnt to infer similarities between terms across ontologies utilizing the entire GFIN. InfAcrOnt builds a term-gene-gene network which comprised ontology annotations and GFIN, and acquires similarities between terms across ontologies through modeling the information flow within the network by random walk. In our benchmark experiments on sub-ontologies of GO, InfAcrOnt achieves a high average area under the receiver operating characteristic curve (AUC) (0.9322 and 0.9309) and low standard deviations (1.8746e-6 and 3.0977e-6) in both human and yeast benchmark datasets exhibiting superior performance. Meanwhile, comparisons of InfAcrOnt results and prior knowledge on pair-wise DO-HPO terms and pair-wise DO-GO terms show high correlations.
\end{abstract}

Conclusions: The experiment results show that InfAcrOnt significantly improves the performance of inferring similarities between terms across ontologies in benchmark set.

Keywords: Biomedical ontology, Term similarities, Random walk, Information flow

\section{Background}

Bio-ontology has drawn more and more attention in the standardization of terminology [1-3], functional annotation of molecules and so on [4-7]. Especially, the relationships between terms of an ontology play an important role in clustering gene expression data for yielding biologically

\footnotetext{
* Correspondence: biofomeng@hotmail.com; huyang@hit.edu.cn

${ }^{\dagger}$ Equal contributors

${ }^{1}$ College of Bioinformatics Science and Technology, Harbin Medical University, Harbin 150081, People's Republic of China

${ }^{5}$ School of Life Science and Technology, Harbin Institute of Technology, Harbin 150088, People's Republic of China

Full list of author information is available at the end of the article
}

meaningful gene clusters [8], prioritizing disease genes for predicting novel disease-causing genes and etc. [9-11].

Nowadays, over 300 biomedical ontologies have been manually curated $[12,13]$. These ontologies are established for describing different types of characteristics of molecules, such as participation in biological processes (BP), induction of diseases, and so on. As the wide application of relationships in single ontology, relationships between terms across ontologies would significantly increase interoperability between molecules in multiple aspects and enable new intelligent bioinformatics applications [14]. 
Gene Ontology (GO) is the earliest and most frequently used ontology, which contains three sub-ontologies (categories) describing molecular function (MF), BP and cellular component (CC) of genes and gene products (Fig. 1). Intra-relationships between terms of each sub-ontology have been manually curated [15] and quantitatively measured [16-19] for dozens of years. By contrast, less attention has been paid to interrelationships between terms of the tree sub-ontologies. Although several methods have been developed to calculate similarities between terms across these sub-ontologies [20-22], it remains a challenge to achieve high reliability.

Since GO has been widely utilized to annotate genes and gene products of various organisms [5], relationships between its terms can also be reflected by their annotated genes. Accordingly, three state-of-art algorithms were designed to identify term relationships among the three sub-ontologies, which include Association Rule Mining (ASR) method [20], Vector Space Model (VSM) method [21], and Cross-Category Gene Ontology Measurement (CroGO) method [22, 23]. ASR method was initially designed to identify products frequently bought together [24]. It was introduced to calculate similarity between terms across sub-ontologies based on the frequency of their annotated gene sets [20]. Subsequently, inter-relationships identified by ASR method across GO's three sub-ontologies were integrated into GO as a complement [25]. VSM method describes each GO term as a vector of genes based on a given annotation database [21]. Then the relationships between terms can be measured by the cosine of their corresponding vectors. Both ASR and VSM methods assume genes are independent and ignore the functional interactions between genes which actually contain valuable information about their corresponding terms. Gene functional interaction network (GFIN) is the widely accepted source of gene interactions at present [26-29]. CroGO utilizes GFIN to enhance its power for the calculation of similarity between terms [22]. It benefits from the additional information stored within gene interaction network which implicates correlations of genes' annotation terms. However, CroGO calculates the similarity between terms only through considering interactions between their annotated genes, but ignores the connectivity among gene nodes of the network. All of these three methods were designed and validated for measuring similarities between terms across GO's three sub-ontologies. They should have the potential to be applied on ontologies built after GO such as Disease Ontology (DO) (Kibbe et al. 2015) and Human Phenotype Ontology (HPO) [30]. However, little work has been done on this aspect.

In this study, we proposed a new method InfAcrOnt to calculate similarities between terms across ontologies utilizing the entire GFIN. In our model, a weighted term-gene-gene network (WTGGN) is created by combining gene annotations and GFIN. Then the information flow in the network is modeled by a random walk $[31,32]$ to calculate term similarities. The method has been validated with experiments on multiple ontologies including DO and HPO.

\section{Methods}

InfAcrOnt has four steps to measure similarities between terms across different ontologies (Fig. 2). First, the weight of term-gene pair was defined. Each of the term-gene pairs was got from a functional annotation of gene. We also define weight of each term in the ontologies. Second, we built a WTGGN based on the weighted term-gene pairs and weighted gene interactions from GFIN. Third, each term was represented as a vector of genes through modeling information flow in

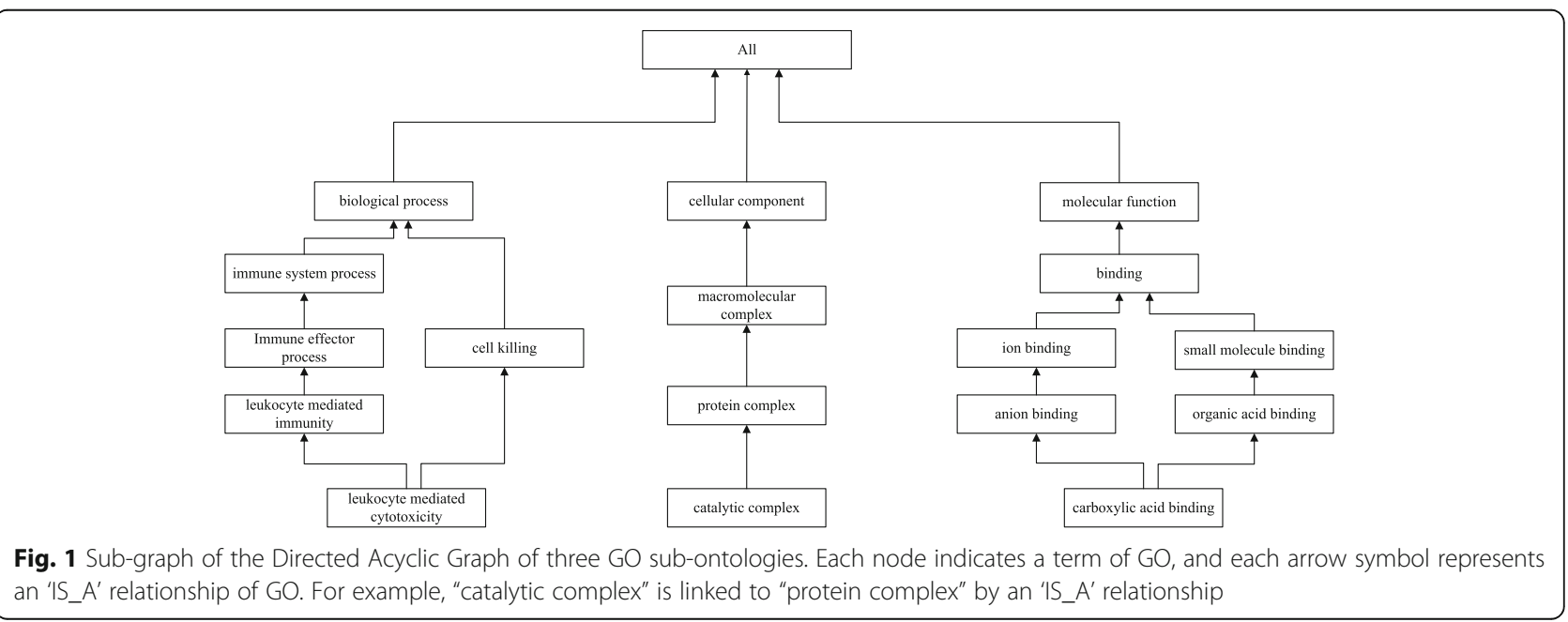



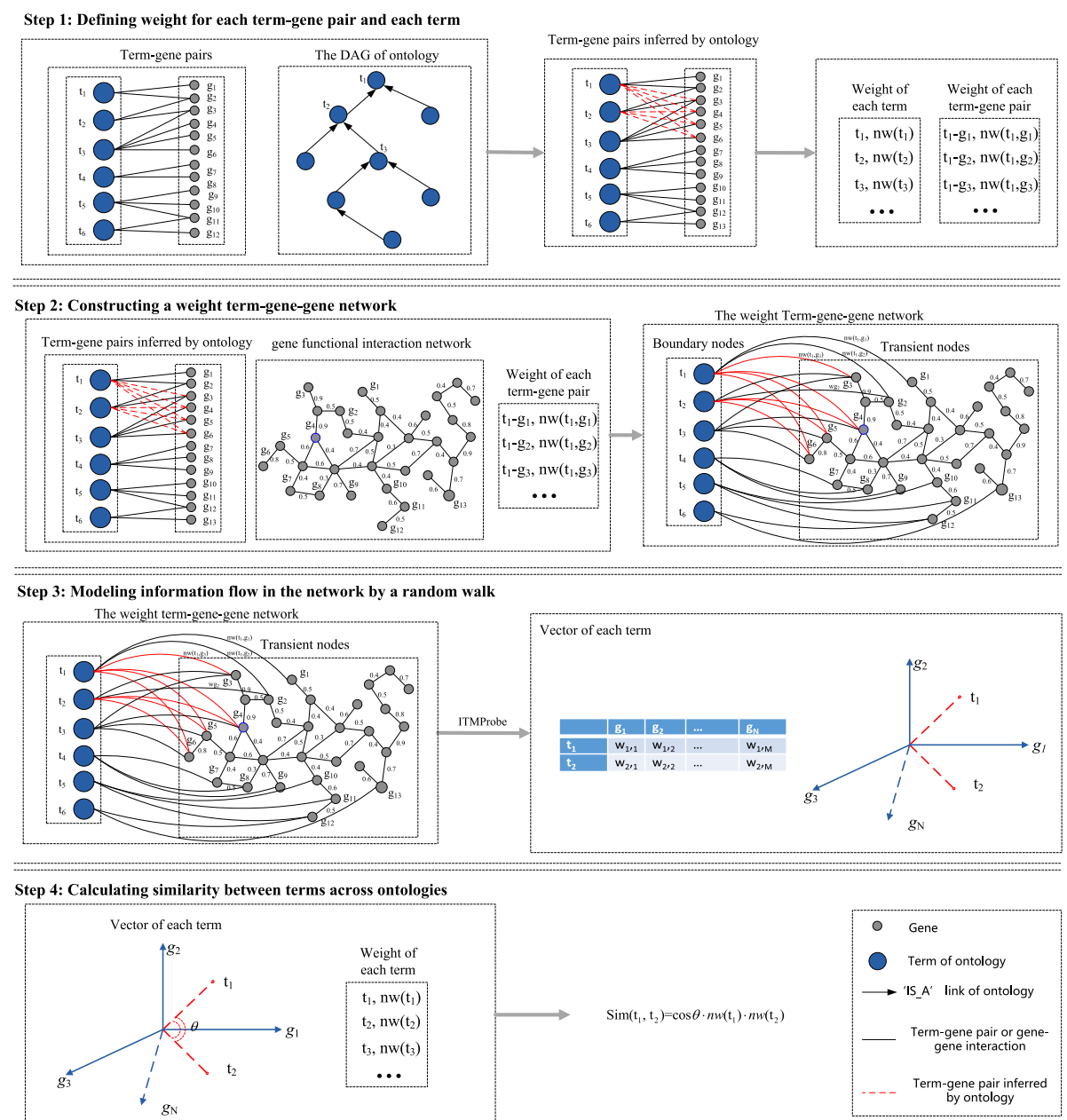

Fig. 2 Overview of InfAcrOnt demonstrating the basic ideas of measuring similarity between terms across ontologies

the WTGGN by random walk. The dimension of vector equals to the number of genes in the network. Fourth, we calculated cosine between vectors and adjust the value with the weight of terms. The results are used as similarities between terms.

\section{Step 1: Defining weight for each term-gene pair and each term}

Ontology annotations provide functional annotations for each gene. Each entry of annotations can be extracted as a term-gene pair. To construct a WTGGN, we define the weight of term-gene pair according to the importance of the gene to a term-gene pair in Eq. 1 , which is inversely proportional to the total number of terms related to gene. Assuming a gene is annotated with only one term, this term-gene relationship should be very important for the WTGGN. On the contrary, if a gene is annotated with multiple terms, the importance of each of these term-gene relationships should be divided equally.

$$
\mathrm{w}\left(\mathrm{t}_{\mathrm{i}}, \mathrm{g}_{\mathrm{j}}\right)=-\log _{2} \frac{n_{j}}{N_{T}}
$$

where $n_{j}$ represents the number of terms associated with the gene $g_{j}, N_{T}$ represents the number of all the annotation terms. Then the weight of each term-gene pair is normalized with Eq. 2.

$$
\mathrm{nw}\left(\mathrm{t}_{\mathrm{i}}, \mathrm{g}_{\mathrm{j}}\right)=\frac{w\left(\mathrm{t}_{\mathrm{i}}, \mathrm{g}_{\mathrm{j}}\right)-w t g_{\min }}{w g_{\max }-w t g_{\min }},
$$

where $w t g_{\min }$ and $w t g_{\max }$ are the minimum and maximum weights of term-gene pairs, respectively.

In an ontology, terms are stored as nodes in a Directed Acyclic Graph (DAG) which are connected with 'IS_A' relationship (Fig. 1). According to the set inclusion relation by 'IS_A' relationships [33], if a gene is annotated by a term, then the gene is also annotated by its ancestors. Therefore, terms in the higher level of the DAG can annotate more genes which lead to shallow annotation [34]. Theoretically, the depth of a term in DAG should 
be inversely proportional to the number of the genes it annotates. To avoid this problem we assign a weight to each term. The weight of term is defined in Eq. 3 in which terms with fewer annotated genes are assigned to relatively higher weight.

$$
\mathrm{w}\left(\mathrm{t}_{\mathrm{i}}\right)=-\log _{2} \frac{n_{g}}{N_{G}},
$$

where $n_{g}$ represents the number of genes annotated by term $t_{i}, N_{G}$ represents the number of all annotated genes. Then the weight of each term is normalized between 0 and 1 with Eq. 4 .

$$
\mathrm{nw}\left(\mathrm{t}_{\mathrm{i}}\right)=\frac{w\left(\mathrm{t}_{\mathrm{i}}\right)-w t_{\min }}{w t_{\max }-w t_{\min }},
$$

where $w t_{\min }$ and $w t_{\max }$ are the minimum and maximum weights of terms, respectively.

Step 2: Constructing a weighted term-gene-gene network We then construct a WTGGN with weighted term-gene pairs achieved in step1 and weighted gene-gene interaction in GFIN. In this network, there are two types of nodes, term nodes and gene nodes. There are also two types of edges, term-gene edge (term node to gene node) and gene-gene edge (gene node to gene node). Each term-gene edge weight is calculated with Eqs. 1 \& 2 and gene-gene weight is sourced from GFIN. The latter is further normalized with Eq. 5 .

$$
\mathrm{w}\left(\mathrm{g}_{\mathrm{i}}, \mathrm{g}_{\mathrm{j}}\right)=\frac{F I S\left(\mathrm{~g}_{\mathrm{i}}, \mathrm{g}_{\mathrm{j}}\right)-F I S_{\min }}{F I S_{\max }-F I S_{\min }},
$$

where $\operatorname{FIS}\left(g_{\dot{v}} g_{j}\right)$ represents functional interaction score between genes $g_{i}$ and $g_{j}$ from GFIN, FIS $S_{\text {min }}$ and $F I S_{\text {max }}$ are the minimum and maximum weights of gene-gene edges, respectively.

The WTGGN contains all necessary information for the calculation of similarities between terms across ontology. This information involves term-gene pairs of ontology annotations and gene-gene interactions of GFIN. In other words, term nodes can be connected by interactions between their annotated gene nodes and intermediate gene nodes in the network, which provides a potential possibility to calculate term similarity more comprehensively.

\section{Step 3: Modeling information flow in the network by a random walk}

Three models have been designed for modeling information flow by a random walk with damping in the network, such as absorbing, emitting and channel models $[35,36]$. The random walk starts from source nodes and terminates either by dissipation or by reaching a sink node. Source nodes and sink nodes are boundary nodes while others are transient nodes. Unlike the classical random walk, these models allow the walker to dissipate or damp at each step under a certain probability. Each walk, if not dissipated, simulates a possible information path from source node to sink node. Absorbing model assigns nodes the random walk ends at, emitting model assigns nodes the random walk starts from and channel model integrates both absorbing and emitting models for directed information flow.

Information Transduction Module (ITM) Probe [37] program has implemented all of these three models. It outputs the expected number of visits to each transient and sink node by random walker originated from every node. The ITM takes an undirected network as input, for each source node it searches for a path to sink nodes under a given dissipation rate. Smaller dissipation rate allows random walks to explore nodes farther to the source while larger dissipation rate evaporates most walks more quickly. In channel model, dissipation rate controls how much a random walk can deviate from the shortest path from sources to sinks. The expected number of visits from the transient nodes to source nodes in the network are scored and returned in terms of the weights by ITM Probe.

Channel model is applied on our WTGGN by ITM Probe. All genes in the network are transient nodes. To access the weight of each gene for a given term, we specified the term as the source node and sink node based on the network. Based on this method, a term could be represented as a weighted vector. Each dimension of the vector is the weight score of a gene to the term. Through random walk in the channel model, the connectivity of the entire network of GFIN can be fully utilized.

Here, the damping factor equals 0.85 according to the previous study [35]. Assuming $N$ genes exist in the WTGGN, each term can be represented as $\mathrm{N}$-dimension vector based on channel model through the ITM Probe. For a given term $t_{1}$, the weighted vector can be described as:

$$
W V_{\mathrm{t}_{\mathrm{i}}}=\left\{w_{i, 1}, w_{i, 2, \cdots}, w_{i, N}\right\},
$$

where $W V_{t i}$ means a weighted vector of $t_{i}$, and $w_{i, j}$ represents the weight score of $t_{i}$ on the $j$ th dimension.

\section{Step 4: Calculating similarities between terms across ontologies}

Then we define the similarity between term $t_{1}$ and $t_{2}$ as following:

$$
\operatorname{Sim}\left(t_{\mathrm{i}}, t_{j}\right)=\cos (\theta) \cdot n w\left(t_{i}\right) \cdot n w\left(t_{j}\right)
$$




$$
\frac{\cos (\theta)=\sum_{n=1}^{N} w_{i, n} \cdot w_{j, n}}{\sqrt{\sum_{n=1}^{N} w_{i, n}^{2}} \sqrt{\sum_{n=1}^{N} w_{j, n}^{2}}}
$$

where the cosine of the vectors of $t_{i}$ and $t_{j}$ is the similarity between terms. The vectors of terms were obtained based on step 3. $n w\left(t_{i}\right)$ and $n w\left(t_{j}\right)$ represent the normalized weight of term $t_{i}$ and $t_{j}$, which could be calculated based on eqs. $3 \& 4$. Here, $n w\left(t_{i}\right)$ and $n w\left(t_{j}\right)$ is used to avoid shallow annotation. The corresponding algorithm was described in the Additional file 1.

\section{Results}

Performance evaluation of calculating similarities of pair-wise BP-MF terms

A benchmark set for human has been built by extracting similar pair-wise BP-MF terms in a previous study [22]. Taken pairs of the benchmark set as our positive group (PG), we get random pairs as a negative group (NG). Then the similarity score of PG and NG was calculated to evaluate the performance of existing methods. e.g. The performance of InfAcrOnt should be superior if the similarity score of the PG can be prioritized at the top.

Pair-wise terms of the benchmark set were generated based on their co-occurrence enzymes [25]. On the one hand, BP terms are also defined as the name of metabolic pathways, each of which is associated with several enzymes. On the other hand, MF terms can also be linked to enzymes with the official GO translations [38, 39]. As a result, 80 pairs of BP-MF terms associated with common enzymes based on HumanCyc [40] were obtained for human as PG. Then 10 times (800 pairs) of benchmark set were obtained randomly as a NG. Here each term of random pairs is selected from the terms with annotated genes.

To calculate similarity of term pairs of PG and NG, we need to construct a WTGGN for BP-MF terms and their annotated genes. GO [15] was downloaded from open source repositories (Table 1) which provided manually curated 'IS_A' relationships between terms [33]. Currently, a total of 12,174 'IS_A' relationships between 9988 MF terms and 54,502 'IS_A' relationships between $28,245 \mathrm{BP}$ terms are included in these ontologies. GO annotations (GOA) of human genes were accessed from GO Consortium (Table 1). Each entry of annotation of GOA was tagged with a GO evidence code. An annotation with the evidence code 'IEA' means it is nonexperimental annotation without confirmed by a human annotator. After removing 'IEA' annotations, $3217 \mathrm{MF}$ terms and 9032 BP terms are used to annotate 14,435 human genes which generate 132,984 annotations were obtained. To the best of our knowledge, HumanNet [29] is the latest and most frequently used GFINs for human.
Table 1 Data sources used for identifying novel relationships across ontologies

\begin{tabular}{ll}
\hline Data source & Web site \\
\hline GO & http://geneontology.org/page/download-ontology \\
GOA for & http://geneontology.org/gene-associations/ \\
yeast & gene_association.sgd.gz \\
GOA for & http://geneontology.org/gene-associations/ \\
human & gene_association.goa_ref_human.gz \\
YeastNet & http://www.inetbio.org/yeastnet/ \\
HumanNet & http://www.functionalnet.org/humannet/ \\
HPO \& HPOA & http://human-phenotype-ontology.github.io/ \\
DO & http://disease-ontology.org/ \\
DOA & http://www.bio-annotation.cn/gene2function/ \\
PubMedA & http://www.bio-annotation.cn/ARSSIC \\
\hline
\end{tabular}

Currently, HumanNet contains 476,399 interactions among 16,243 human genes. Using GO, GOA and HumanNet a WTGGN for BP-MF terms and their annotated genes was constructed based on step 1 and step 2 of the 'Methods' section. Then the similarity of term pairs of PG and NG was calculated based on step 3 and step 4 of the 'Methods' section.

The performances of existing methods are assessed by drawing a receiver operating characteristic (ROC) curve. We can get true-positive (TP), false-positive (FP), truenegative (TN), and false-negative (FN) using various similarity scores of PG and NG as threshold. Then the curve is created by plotting the true positive rate (TPR $=(\mathrm{TP}) /(\mathrm{TP}+\mathrm{FN}))($ or Sensitivity) against the false positive rate $(\mathrm{FPR}=1-(\mathrm{TN}) /(\mathrm{TN}+\mathrm{FP}))$ (or 1 -Specificity) at various threshold settings. The area under the ROC curve (AUC) showed the performance of each method for distinguishing PG from NG. Figure 3a shows a ROC curve of the existing method based on our PG and a NG. The corresponding AUCs by the CroGO, VSM, ASR, and InfAcrOnt methods are 0.6539, 0.7674, 0.7659, and 0.9330 respectively. ASR and VSM methods are the two classical approaches. The similar ROCs of these two methods show that the performances of these two methods are almost the same. Although CroGO method introduced the interactions between genes, it did not perform well. This may be caused by the fact that the connectivity between genes through the GFIN wasn't be utilized. Fortunately, the entire GFIN was incorporated in the InfAcrOnt method. And the significantly higher AUC (0.9330) validates that our method helps to enhance the true positive rate and reduces the false positive rate.

The experiment was iterated 100 times based on 100 random NGs. The AUCs of 100 iterations are shown in Fig. 3b. The average AUCs of the CroGO, VSM, ASR, and InfAcrOnt methods are 0.6509, 0.7721, 0.7690, and 0.9322 respectively. And the corresponding standard deviations (SDs) of these AUCs are 1.5699e-4, 3.9732e-5, 

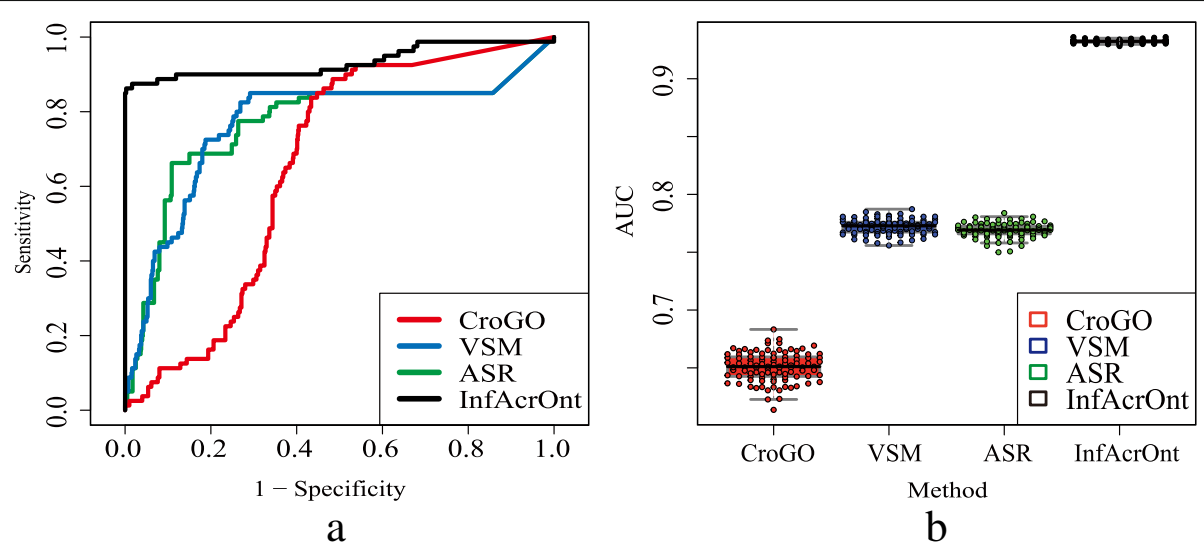

Fig. 3 ROC analysis of the benchmark set and random sets for human. a ROC curves for the experimental results on the benchmark set and a random set for human. It shows 1-specificity versus sensitivity of each method for calculating the similarities of terms across BP and MF. b Average of AUC for 100 iterators for human

$3.5278 \mathrm{e}-5$, and $1.8746 \mathrm{e}-6$ respectively. In comparison with other methods, AUC is improved more than 0.15 by InfAcrOnt. The highest AUC and lowest SD of InfAcrOnt shows a significant advantage of our method.

Peng et al. also provide another benchmark set of similar pair-wise BP-MF terms for yeast [22]. Then the similar experiment was done on yeast. The set was generated based on their co-occurrence enzymes [25]. Finally, 175 pairs of BP-MF terms related with common enzymes by YeastCyc [41] were obtained for yeast as PG, and corresponding 1750 random pairs were obtained as a NG. The WTGGN for calculating term pair similarity was built based on GO, GOA for yeast, and YeastNet [28]. After removing 'IEA' annotations, $1676 \mathrm{MF}$ terms and $2655 \mathrm{BP}$ terms are used to annotate 6332 yeast genes which generate 26,488 annotations were obtained. YeastNet [28] is the latest and most frequently used GFINs for yeast. It includes 362,421 interactions between 5809 yeast genes.

The results of benchmark set for yeast are shown in Additional file 2. According to this figure, the AUCs of one of our experiments for yeast by the CroGO, VSM, ASR, and InfAcrOnt methods are 0.6689, 0.7640, 0.7660, and 0.9307 respectively. The AUCs of 100 iterations for yeast are shown in Additional file 2. The average AUCs of the CroGO, VSM, ASR, and InfAcrOnt methods for yeast are 0.6546, 0.7608, 0.7664 , and 0.9308 respectively. And the corresponding SDs of these AUCs are 4.3988e-5, 2.1204e-5, 1.6300e-5 and $3.0977 \mathrm{e}-6$ respectively. These results show the consistency in both human and yeast. This indicates that the advantage of InfAcrOnt in calculating similarity of BP-MF terms is stable and reliable.

\section{Performance evaluation of calculating similarities of pair-wise DO-HPO terms}

To show InfAcrOnt's ability to work on ontologies other than GO's 3 sub-ontologies, we calculated similarities of pair-wise DO-HPO terms. The similarity of DO-HPO term pairs can also be calculated based on prior knowledge in HPO project [42] by Term Frequency Inverse Document Frequency (TF-IDF) [43]. Theoretically, similarity score between terms based on genes should be consistent with this based on phenotypes. Therefore, we calculated the Pearson correlation coefficient between InfAcrOnt similarity score and TF-IDF similarity score to evaluate the performance of InfAcrOnt.

A WTGGN for DO-HPO terms and their annotated genes was built by DO, HPO, DO Annotations (DOA), HPO Annotations (HPOA), and HumanNet (Table 1). Then the similarities of pair-wise DO-HPO terms were calculated by InfAcrOnt based on the WTGGN. DO [44] and HPO [30] were downloaded from open source repositories (Table 1 ) which provided manually curated 'IS_A' relationships between terms [33]. Currently, 15,459 'IS_A' relationships between 11,673 HPO terms and 7124 'IS_A' relationships of 6920 DO terms are included in these ontologies. DOA [10] were sourced from the annotations of GeneRIF [45]. After removing duplication, 98,008 associations between 2576 diseases and 9991 genes were obtained. HPOA of human genes were accessed from the HPO project [42] which provided annotated genes relative to human phenotype. Currently, it contains 120,890 associations between 5838 terms and 3496 genes. HumanNet has been accessed in 3.1 section.

HPO project [42] parsed textual descriptions of each disease in the Clinical Synopsis section of OMIM entry. And the phenotypes of the textual descriptions were extracted and organized into HPO. Diseases of OMIM entries were mapped to DO terms based on crossreference $[44,46]$. Notably, a phenotype occurred in textual descriptions of a disease only shows a text relevance between the phenotype and the disease. Thus we need to quantify this text relevance. To this end, we 
constructed a n-by-m matrix where $\mathrm{N}$ was the number of DO terms and M was the number of HPO terms. The ( $i$ th, $j$ th) element of the matrix was valued with the number of occurrences of $j$ th row phenotype in the textual descriptions of $i$ th disease. Subsequently, we applied TF-IDF [43], a typical model for quantifying text relevance, to calculate the similarity between HPO terms and DO terms based on the matrix.

Figure 4 shows the correlation between InfAcrOnt similarity score and TF-IDF similarity score (Pearson correlation, $\left.\gamma^{2}=0.1158 p=2.2 \mathrm{e}-16\right)$. The high correlation validated the good performance of InfAcrOnt in calculating the similarity of DO-HPO terms. To further test the performance of the proposed method, InfAcrOnt was compared with the state-of-art methods including ASR, VSM, and CroGO. The comparison results are shown in Fig. $4 \mathrm{~b}$ and Additional file 3. The similarity based on the ARS method accessed the lowest correlation with the TF-IDF similarity (Pearson correlation, $\gamma^{2}=0.0163 p=0.0062$ ), which is shown in Fig. $4 \mathrm{~b}$ and Additional file 3 In comparison, the TF-IDF similarity is more correlated with the similarity based on the CroGO method (Pearson correlation, $\gamma^{2}=0.1015 \mathrm{p}=2.2 \mathrm{e}-16$; Fig. 4b and Additional file 3), the VSM method (Pearson correlation, $\gamma^{2}=0.1083 p=2.2 e-16$; Fig. $4 \mathrm{~b}$ and Additional file 3). As expected, similar terms could be identified based on existing methods and prior knowledge in HPO project simultaneously. In comparison with these state-ofart methods, InfAcrOnt similarity achieves the most correlation with prior knowledge.

\section{Performance evaluation of calculating similarities of pair-wise DO-BP terms}

To show InfAcrOnt's ability to calculate similarity between terms across GO's sub-ontologies and other ontologies, we applied our method in DO-BP terms. An alternative way to calculate the similarity of DO-BP term pairs is based on prior knowledge in PubMed [47] by the Extensional Mutual Information (EMI) method [48]. Then the correlation between InfAcrOnt similarity score and EMI similarity score was utilized to evaluate the performance of InfAcrOnt.

A WTGGN for DO-GO terms and their annotated genes was constructed by DO, GO, DOA, GOA and HumanNet. All of these data have been accessed in 3.1 and 3.2 sections. Then the similarities of pair-wise DO-BP terms were calculated by InfAcrOnt based on the WTGGN.

Literature of PubMed documents DO terms and GO terms in its title and abstract. Two terms occur in a literature is defined as a co-occurrence relationship between them [48]. These co-occurrence relationships can be quantified as the similarity of DO-BP term pairs. One of the most frequently used algorithm to do this is EMI by Wren et al. [48]. Here we downloaded the cooccurrence relationships of DO-BP term pairs in PubMed from the previous study [9], and then calculated the EMI similarity of DO-BP term pairs.

Figure 5 shows the correlation between InfAcrOnt similarity score and EMI similarity score (Pearson correlation, $\gamma^{2}=0.2429 p=2.2 \mathrm{e}-16$ ). The high correlation validated the good performance of InfAcrOnt in calculating the similarity of DO-BP terms. To further test the performance of the proposed method, InfAcrOnt was compared with ASR, VSM, and CroGO. The comparison results are shown in Fig. 5b and Additional file 4. As expected, the results show that EMI similarity is also positive correlated with the similarity based on the CroGO method (Pearson correlation, $\gamma^{2}$ $=0.0296 \mathrm{p}=2.2 \mathrm{e}-16$; Fig. $5 \mathrm{~b}$ and Additional file 4), the VSM method (Pearson correlation, $\gamma^{2}=0.2092 \mathrm{p}=2.2 \mathrm{e}-16$; Fig. 5b and Additional file 4), the ASR method (Pearson correlation, $\gamma^{2}=0.0605 p=2.2 e-16$; Fig. 5b and Additional file 4). In comparison, the similarity based on the InfAcrOnt method is the most relevant with the EMI similarity.

\section{Case studies: diabetes mellitus, alzheimer's disease, and neuroblastoma related biological process}

To further indicate our method in identifying diseaserelated BP, case studies of Diabetes Mellitus (DM), Alzheimer's Disease (AD), and Neuroblastoma were

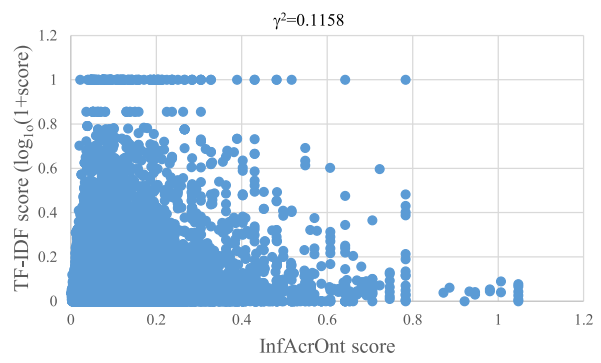

a

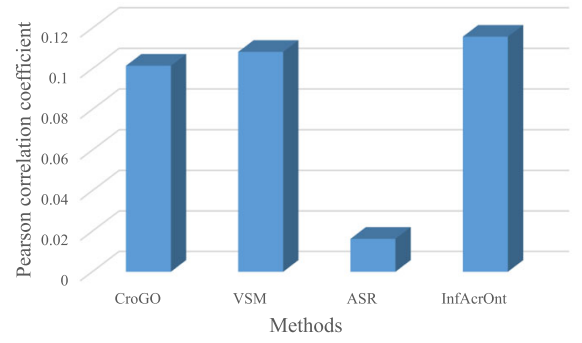

b

Fig. 4 The correlation between the term similarity based on ontology annotations and prior knowledge in HPO project. a The distribution of the similarity scores by InfAcrOnt method. b Pearson Correlation Coefficient between similarity scores based on TF-IDF and other methods 


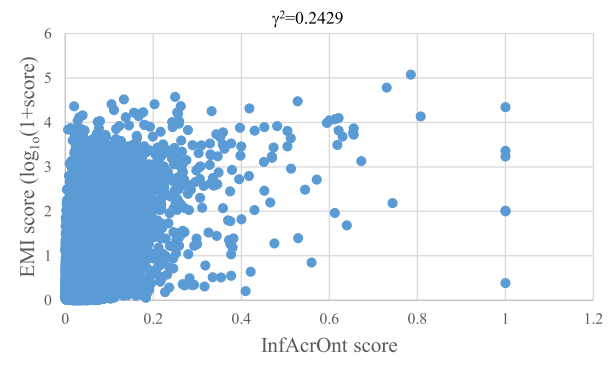

a

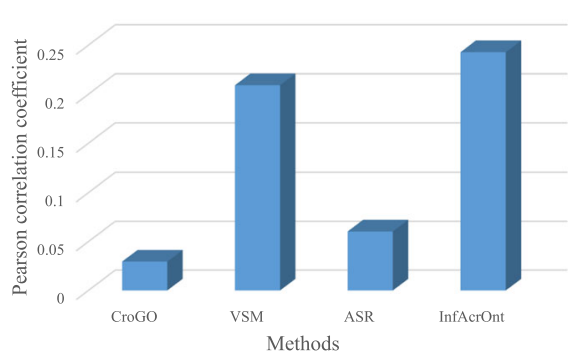

b

Fig. 5 The correlation between the term similarity based on ontology annotations and prior knowledge in PubMed. a The distribution of the similarity scores by InfAcrOnt method. b Pearson Correlation Coefficient between similarity score based on EMI and other methods

examined. The similarity of DO-BP terms was calculated in section 3.3. Here we ranked the BP terms of diseases by the InfAcrOnt similarity score, and then investigated top 5 similar BP terms of these three diseases respectively. Their relationships were manually checked in the published studies and the results were listed in Additional file 5. All of five DM-BP relationships were validated. And four of five AD-BP relationships and four of five neuroblastoma-BP relationships were also validated. For example, DM increases reactive oxygen species (GO:0000302) production [49], DNA replication (GO:0006275) stress is a key element of AD [50]. All of these results indicate that our method can function in identifying potential DO-BP terms.

\section{Discussion}

The importance of the relationship between terms across ontologies had been reflected in the previous researches $[14,51,52]$. However, few of these relationships were manually curated in the existing vocabularies. Currently, methods have been developed for measuring the similarity between terms across ontologies based on term-gene pairs of ontology annotations, which can prioritize these inter-relationships [20-22]. Because of ignoring the connectivity of the GFIN, existing methods were limited for identifying novel relationships. To solve this problem, in this article we devised a new method named InfAcrOnt for improving the performance of calculating the similarity of terms across ontologies by integrating ontology annotations and GFIN through information flow.

The performance of InfAcrOnt was validated very well in calculating similarities of BP-MF term pairs according to the evaluation on two benchmark sets (Fig. 3 and Additional file 2). The two benchmark sets were selected strictly by their common enzymes (see ' 3.1 ' section). Therefore, our method is very suitable for identifying strong relationships. Because two benchmark sets are sourced from human and yeast, respectively, and the experiment was iterated 100 times, the stability of our method was also proved very well.

The superior performance of InfAcrOnt was also validated in calculating the similarity of pair-wise DO-HPO terms (Fig. 4) and pair-wise DO-GO terms (Fig. 5). The high correlations between similarity based on existing methods and similarity based on prior knowledge show that the performance of the ASR, VSM, CroGO, and InfAcrOnt methods are also good for other ontologies besides sub-ontologies of GO. Considering the fluctuation of the performance of the ASR and CroGO (Figs. 4b and 5b) methods, the VSM and InfAcrOnt methods perform better. In comparison with other methods, InfAcrOnt achieves the highest correlation, which means it is the most consistent with prior knowledge.

Over 300 ontologies have been developed in the biomedical domain. The lack of relationships between terms across these ontologies limited the interoperability in term level. Fortunately, InfAcrOnt can function in identifying novel relationships based on ontology annotations and GFIN. Because most of the ontologies were used to annotate genes and GFIN has been constructed $[28,29]$, InfAcrOnt can be used widely for calculating similarities between terms across these ontologies. Furthermore, the case studies validate the method can function in identifying novel relationships.

\section{Conclusions}

In this article, we presented a novel method InfAcrOnt for calculating cross-ontology term similarities using information flow by a random walk. The method mainly focused on taking advantage of the connectivity of the GFIN. To validate its performance, experiments were conducted on InfAcrOnt and state-of-art methods on sub-ontologies of GO and other frequently used ontologies. The highest AUC (0.9322 and 0.9309) and lowest SDs (1.8746e-6 and 3.0977e-6) were achieved for InfAcrOnt in both human and yeast benchmark datasets. 
And the highest correlation were also obtained between similarity score using InfAcrOnt and prior knowledge for DO-HPO (Pearson correlation, $\gamma^{2}=$ $0.1158 \mathrm{p}=2.2 \mathrm{e}-16$ ) and DO-BP (Pearson correlation, $\left.\gamma^{2}=0.2429 \mathrm{p}=2.2 \mathrm{e}-16\right)$ terms. All of these results exhibited the superiority of our method. In the case study, novel identified BPs of DM and AD using InfAcrOnt were verified in recent literatures. Currently, over 300 ontologies without interoperability in term level have been developed in the biomedical domain. Therefore, it is valuable for using InfAcrOnt to mine novel relationships across ontologies.

\section{Additional files}

Additional file 1: Algorithm for measuring term similarities across ontologies. (PDF 193 kb)

Additional file 2: AUC analysis of the benchmark set and random sets for yeast. (PDF $463 \mathrm{~kb}$ )

Additional file 3: The correlation between the term similarity by state-of-art methods and prior knowledge in HPO project. (PDF $145 \mathrm{~kb}$ )

Additional file 4: The correlation between the term similarity by state-of-art methods and prior knowledge in PubMed. (PDF 134 kb)

Additional file 5: Disease-related biological process confirmed by literature mining. (PDF $102 \mathrm{~kb}$ )

\section{Abbreviations}

AD: Alzheimer's disease; ASR: Association rule mining; AUC: Area under the receiver operating characteristic curve; BP: Biological processes; CC: Cellular component; CroGO: Cross-category gene ontology measurement; DM: Diabetes mellitus: DO: Disease ontology; EMI: Extensional mutual information; FN: False-negative; FP: False-positive; FPR: False positive rate; GFIN: Gene functional interaction network; GO: Gene ontology; GOA: GO annotations; HPO: Human phenotype ontology; HPOA: HPO annotations; ITM: Information transduction module; MF: Molecular function; NG: Negative group; PG: Positive group; ROC: Receiver operating characteristic; SDs: Standard deviations; TF-IDF: Term frequency inverse document frequency; TN: True-negative; TP: True-positive; TPR: True positive rate; VSM: Vector space model; WTGGN: Weighted term-gene-gene network

\section{Acknowledgments}

We would like to thank Dr. Jin Chen, associate Professor at University of Kentucky, for invaluable comments and suggestions to the project.

\section{Funding}

The publication costs of this article were supported by the National Natural Science Foundation of China (Grant No. 61502125), Heilongjiang Postdoctoral Fund (Grant No. LBH-Z15179), and China Postdoctoral Science Foundation (Grant No. 2016 M590291). The funders had no roles in study design, data collection and analysis, decision to publish, or preparation of the manuscript.

\section{Availability of data and materials}

The datasets during and/or analyzed during the current study available from the corresponding author on reasonable request.

\section{About this supplement}

This article has been published as part of BMC Genomics Volume 19 Supplement 1, 2017: 16th International Conference on Bioinformatics (InCoB 2017): Genomics. The full contents of the supplement are available online at https://bmcgenomics.biomedcentral.com/articles/supplements/volume-19supplement-1.

\section{Authors' contributions}

LC, MZ, YH conceived and designed the experiments. LC, YJ, HJ, JS, and JP analyzed data. LC and YJ wrote this manuscript. All authors read and approved the final manuscript.

Ethics approval and consent to participate

Not applicable.

\section{Consent for publication}

Not applicable.

\section{Competing interests}

The authors declare that they have no competing interests.

\section{Publisher's Note}

Springer Nature remains neutral with regard to jurisdictional claims in published maps and institutional affiliations.

\section{Author details}

${ }^{1}$ College of Bioinformatics Science and Technology, Harbin Medical University, Harbin 150081, People's Republic of China. ${ }^{2}$ Hospital for Sick Children, Toronto M5G 1X8, Canada. ${ }^{3}$ Department of Information Engineering, Heilongjiang Biological Science and Technology Career Academy, Harbin 150081, People's Republic of China. ${ }^{4}$ School of Computer Science, Northwestern Polytechnical University, Xian 710072, People's Republic of China. ${ }^{5}$ School of Life Science and Technology, Harbin Institute of Technology, Harbin 150088, People's Republic of China.

Published: 19 January 2018

\section{References}

1. Hastings J, de Matos P, Dekker A, Ennis M, Harsha B, Kale N, Muthukrishnan V, Owen G, Turner S, Williams M. The ChEBI reference database and ontology for biologically relevant chemistry: enhancements for 2013. Nucleic Acids Res. 2013:41(D1):D456-63.

2. Schindelman G, Fernandes JS, Bastiani CA, Yook K, Sternberg PW. Worm phenotype ontology: integrating phenotype data within and beyond the $\mathrm{C}$. Elegans community. BMC bioinformatics. 2011;12:32.

3. Smith CL, Goldsmith CA, Eppig JT. The mammalian phenotype ontology as a tool for annotating, analyzing and comparing phenotypic information Genome Biol. 2005;6(1):R7

4. Smith CL, Eppig JT. The mammalian phenotype ontology as a unifying standard for experimental and high-throughput phenotyping data. Mamm Genome. 2012:23(9-10):653-68.

5. Camon E, Magrane M, Barrell D, Lee V, Dimmer E, Maslen J, Binns D, Harte N, Lopez R, Apweiler R. The gene ontology annotation (goa) database: sharing knowledge in uniprot with gene ontology. Nucleic Acids Res. 2004; 32(suppl 1):D262-6

6. Osborne JD, Flatow J, Holko M, Lin SM, Kibbe WA, Zhu LJ, Danila MI, Feng G, Chisholm RL. Annotating the human genome with disease ontology. BMC Genomics. 2009;10(Suppl 1):S6

7. Robinson PN, Kohler S, Bauer S, Seelow D, Horn D, Mundlos S. The human phenotype ontology: a tool for annotating and analyzing human hereditary disease. Am J Hum Genet. 2008;83(5):610-5.

8. Kustra R, Zagdanski A: Incorporating gene ontology in clustering gene expression data. In: 19th IEEE Symposium on Computer-Based Medical Systems (CBMS'06). 2006;555-63.

9. Cheng L, Li J, Hu Y, Jiang Y, Liu Y, Chu Y, Wang Z, Wang Y. Using semantic association to extend and infer literature-oriented relativity between terms. IEEE/ACM Trans. Comput. Biol. Bioinform. 2015;12(6):1219-26.

10. Hu Y, Zhou W, Ren J, Dong L, Wang Y, Jin S, Cheng L: Annotating the function of the human genome with gene ontology and disease ontology. BioMed Research International 2016.

11. Young MD, Wakefield MJ, Smyth GK, Oshlack A. Method gene ontology analysis for RNA-seq: accounting for selection bias. Genome Biol. 2010;11:R14

12. Whetzel PL, Team N. NCBO technology: powering semantically aware applications. J biomed semant. 2013;4(Suppl 1):S8

13. Smith B, Ashburner M, Rosse C, Bard J, Bug W, Ceusters W, Goldberg L, Eilbeck K, Ireland A, Mungall CJ, et al. The OBO foundry: coordinated evolution of ontologies to support biomedical data integration. Nat Biotechnol. 2007;25(11):1251-5. 
14. Soldatova LN, King RD. Are the current ontologies in biology good ontologies? Nat Biotechnol. 2005;23(9):1095-8.

15. Ashburner M, Ball CA, Blake JA, Botstein D, Butler H, Cherry JM, Davis AP, Dolinski K, Dwight SS, Eppig JT. Gene ontology: tool for the unification of biology. Nat Genet. 2000;25(1):25-9.

16. Wang JZ, Du Z, Payattakool R, PS Y, Chen CF. A new method to measure the semantic similarity of $\mathrm{GO}$ terms. Bioinformatics. 2007; 23(10):1274-81.

17. Resnik P: Using information content to evaluate semantic similarity in a taxonomy. arXiv preprint cmp-lg/9511007 1995

18. Lin D. An information-theoretic definition of similarity. In: ICML: 1998; 1998. p. 296-304.

19. Peng JJ, Xue HS, Shao YK, Shang XQ, Wang YD, Chen J. A novel method to measure the semantic similarity of HPO terms. Int J Data Min Bioin. 2017; 17(2):173-88.

20. Kumar A, Smith B, Borgelt C: Dependence relationships between Gene Ontology terms based on TIGR gene product annotations. In. 2008.

21. Bodenreider O, Aubry M, Burgun A. Non-lexical approaches to identifying associative relations in the gene ontology. Pac. Symp. Biocomput.Pac. Symp. Biocomput. 2005:91-102.

22. Peng J, Chen J, Wang Y. Identifying cross-category relations in gene ontology and constructing genome-specific term association networks. BMC Bioinf. 2013;14(2):1.

23. Peng J, Wang $H$, Lu J, Hui W, Wang Y, Shang $X$ : Identifying term relations cross different gene ontology categories. BMC bioinformatics 2017.

24. Borgelt C, Kruse R. Induction of association rules: Apriori implementation. In: Compstat: 2002: Springer; 2002. p. 395-400.

25. Myhre S, Tveit H, Mollestad T, Laegreid A. Additional gene ontology structure for improved biological reasoning. Bioinformatics. 2006;22(16):2020-7.

26. Hamaneh MB, YK Y. Relating diseases by integrating gene associations and information flow through protein interaction network. PLoS One. 2014:9(10):e110936.

27. Cheng L, Shi H, Wang Z, Hu Y, Yang H, Zhou C, Sun J, Zhou M: IntNetLncSim: an integrative network analysis method to infer human IncRNA functional similarity. Oncotarget 2016.

28. Kim H, Shin J, Kim E, Kim H, Hwang S, Shim JE, Lee I: YeastNet v3: a public database of data-specific and integrated functional gene networks for Saccharomyces cerevisiae. Nucleic acids research. 2014;42(Database issue): 731-7.

29. Lee I, Blom UM, Wang PI, Shim JE, Marcotte EM. Prioritizing candidate disease genes by network-based boosting of genome-wide association data. Genome Res. 2011;21(7):1109-21.

30. Robinson PN, Mundlos S. The human phenotype ontology. Clin Genet. 2010;77(6):525-34.

31. Wu Q, Ng MK, Ye Y, Li X, Shi R, Li Y. Multi-label collective classification via Markov chain based learning method. Knowl-Based Syst. 2014;63(3):1-14.

32. Wu Q, Ng MK, Ye Y. Markov- Miml : a Markov chain-based multi-instance multi-label learning algorithm. Knowl. Inf. Syst. 2013;37(1):83-104.

33. Smith B, Ceusters W, Klagges B, Köhler J, Kumar A, Lomax J, Mungall C, Neuhaus F, Rector AL, Rosse C. Relations in biomedical ontologies. Genome Biol. 2005;6(5):R46.

34. J. L S, V S, A. P, E. G, J. M M, M-C LA, F. J C, A R. Correlation between gene expression and GO semantic similarity. IEEE/ACM Trans. Comput. Biol. Bioinform. 2005;2(4):330-8.

35. Stojmirović A, Y-K Y. Information flow in interaction networks II: channels, path lengths, and potentials. J Comput Biol. 2012;19(4):379-403.

36. Stojmirovic A, YK Y. Information flow in interaction networks. J. Comput. Mol. Cell Biol. 2007;14(8):1115-43.

37. Stojmirović A, Y-K Y. ITM probe: analyzing information flow in protein networks. Bioinformatics. 2009;25(18):2447-9.

38. Hill DP, Davis AP, Richardson JE, Corradi JP, Ringwald M, Eppig JT, Blake JA Program description: strategies for biological annotation of mammalian systems: implementing gene ontologies in mouse genome informatics. Genomics. 2001;74(1):121-8.

39. Camon EB, Barrell DG, Dimmer EC, Lee V, Magrane M, Maslen J, Binns D, Apweiler R. An evaluation of GO annotation retrieval for BioCreAtlvE and GOA. BMC Bioinf. 2005;6(1):1

40. Caspi R, Foerster H, Fulcher CA, Hopkinson R, Ingraham J, Kaipa P, Krummenacker M, Paley S, Pick J, Rhee SY. MetaCyc: a multiorganism database of metabolic pathways and enzymes. Nucleic Acids Res. 2006; 34(suppl 1):D511-6.
41. Caspi R, Foerster H, Fulcher CA, Kaipa P, Krummenacker M, Latendresse M, Paley S, Rhee SY, Shearer AG, Tissier C. The MetaCyc database of metabolic pathways and enzymes and the BioCyc collection of pathway/genome databases. Nucleic Acids Res. 2008;36(suppl 1):D623-31.

42. Köhler S, Doelken SC, Mungall CJ, Bauer S, Firth HV, Bailleul-Forestier I, Black GC, Brown DL, Brudno M, Campbell J. The human phenotype ontology project: linking molecular biology and disease through phenotype data. Nucleic Acids Res. 2014;42(D1):D966-74.

43. Salton G, Wong A, Yang CS. A vector space model for automatic indexing Commun ACM. 1975;18(11):273-80.

44. Kibbe WA, Arze C, Felix V, Mitraka E, Bolton E, Fu G, Mungall CJ, Binder JX, Malone J, Vasant D, et al. Disease ontology 2015 update: an expanded and updated database of human diseases for linking biomedical knowledge through disease data. Nucleic Acids Res. 2015;43(Database issue):D1071-8.

45. Osborne JD, Lin S, Kibbe W, Zhu L, Danila M, Chisholm RL. GeneRIF is a more comprehensive, current and computationally tractable source of gene-disease relationships than OMIM. Bioinf. Core, Northwes Univ Tec Rep. 2007;

46. Cheng L, Wang G, Li J, Zhang T, Xu P, Wang Y. SIDD: a semantically integrated database towards a global view of human disease. PLoS One. 2013;8(10):e75504.

47. Coordinators NR: Database Resources of the National Center for Biotechnology Information. Nucleic Acids Res. 2013;41(Database issue): D8-D20.

48. Wren JD. Extending the mutual information measure to rank inferred literature relationships. BMC Bioinf. 2004;5:145.

49. Santos MC, Louzada RA, Souza EC, Fortunato RS, Vasconcelos AL, Souza KL, Castro JP, Carvalho DP, Ferreira AC. Diabetes mellitus increases reactive oxygen species production in the thyroid of male rats. Endocrinology. 2013; 154(3):1361-72

50. Yurov YB, Vorsanova SG, lourov IY. The DNA replication stress hypothesis of Alzheimer's disease. TheScientificWorldJOURNAL. 2011;11:2602-12.

51. Sanchez D, Sole-Ribalta A, Batet M, Serratosa F. Enabling semantic similarity estimation across multiple ontologies: an evaluation in the biomedical domain. J Biomed Inform. 2012:45(1):141-55.

52. Kahn CE, Jr.: Integrating ontologies of rare diseases and radiological diagnosis. J. Am. Med. Inform. Assoc. : JAMIA 2015, 22(6):1164-1168.

\section{Submit your next manuscript to BioMed Central and we will help you at every step:}

- We accept pre-submission inquiries

- Our selector tool helps you to find the most relevant journal

- We provide round the clock customer support

- Convenient online submission

- Thorough peer review

- Inclusion in PubMed and all major indexing services

- Maximum visibility for your research

Submit your manuscript at www.biomedcentral.com/submit
) Biomed Central 